\title{
A Novel De Novo Missense Mutation in HNF4A Resulting in Sulfonylurea-Responsive Maturity- onset Diabetes of the Young
}

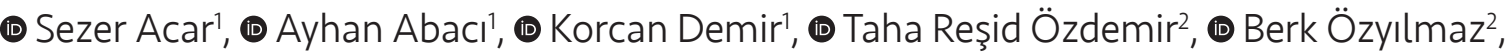 \\ (1) Ece Böber ${ }^{1}$ \\ 'Dokuz Eylül University Faculty of Medicine, Department of Pediatrics, Division of Pediatric Endocrinology, İzmir, Turkey \\ 2University of Health Sciences, İzmir Tepecik Training and Research Hospital, Clinic of Medical Genetics, İzmir, Turkey
}

\begin{abstract}
Maturity-onset diabetes of the young (MODY) is a monogenic form of diabetes, with autosomal-dominant inheritance, which usually develops before 25 years of age. MODY is classically caused by a heterozygous mutation of genes known to affect insulin production or secretion. Heterozygous inactivating hepatocyte nuclear factor 4A (HNF4A) mutations, one of the rare subtypes of MODY, cause impaired insulin secretion and subsequent glucose intolerance especially in adolescence. Conversely, HNF4A mutations are also known to be associated with macrosomia and hyperinsulinemic hypoglycemia in newborns. Herein, we report a rare cause of diabetes resulting from a novel heterozygous mutation in the HNF4A gene. In conclusion, genetic testing should be considered in order to establish an accurate diagnosis and provide an opinion in determining the appropriate type of treatment.

Keywords: Maturity-onset diabetes of the young Type I, macrosomia, HNF4A, monogenic diabetes, child
\end{abstract}

\section{Introduction}

Maturity-onset diabetes of the young (MODY) is a monogenic subgroup of diabetes mellitus characterized by autosomal dominant inheritance, non-insulin diabetes onset usually before 25 years of age and decreased insulin production or secretion response to glucose. At least 13 different genes have been reported to be associated with MODY to date (1). Approximately $1-2 \%$ of patients with diabetes have a monogenic type (2). The inactivating mutations in the nuclear transcription factor 1 homeobox A (HNFTA), the hepatocyte nuclear factor 4 homeobox
(HNF4A) and the glucokinase (GCK) are the most common causes of MODY (3). Other genes associated with MODY are infrequently detected: HNFIB, IPF, NEUROD, PDXI, KLF11, CEL, PAX4, BLK, ABCC8 and KCNJ11 (3). While the heterozygous inactivating mutations in the CCK gene lead to asymptomatic mild fasting hyperglycemia, mutations in the genes of HNFTA and HNF4A lead to progressive failure in insulin secretion and worsening of glucose tolerance with age (4). HNF4A is a member of the steroid/thyroid hormone receptor superfamily and plays a major role in glucose stimulated insulin secretion. Homozygous HNF4A mutation is lethal at the early embryonic stage (5). However, 
heterozygous HNF4A mutations have a Janus effect on glucose metabolism, which leads to either macrosomia and hyperinsulinemic hypoglycemia during infancy or MODY Type I in adulthood (6). The mutations of the genes involved in MODY are typically inherited from affected parents. However, a few de novo mutations have also been reported to date $(4,7)$. In this study, we report on an interesting patient with MODY Type I that resulted from a novel and de novo mutation in the HNF4A gene.

\section{Case Report}

A 14-year-old girl was referred to our outpatient clinic due to fatigue and polyuria; and hyperglycemia was detected afterwards. She was born full term after an uneventful pregnancy with a birth weight of 5.500 gr [4.9 standard deviation (SD) score]. Her parents were healthy and there was no consanguinity between them. The family history revealed no diabetes. Physical examination of the case revealed a height of $163 \mathrm{~cm}$ (SD score 0.29), weight of $64.7 \mathrm{~kg}$ (SD score 1.2) and body mass index (BMI) of $24 \mathrm{~kg} / \mathrm{m}^{2}$ (SD score 1.2). Acanthosis nigricans or stria was not found. A puberty examination according to the Tanner scale was stage 5 and she had a regular pattern of menstrual periods. On admission, laboratory analyses showed hyperglycemia, a relatively low level of C-peptide, elevated glycated hemoglobin (HbA1c), a low level of triglycerides and negative autoantibodies regarding diabetes (Table I). Urine analysis revealed 2+ glycosuria and no ketosis. The parents had normal fasting blood glucose

\begin{tabular}{|l|l|l|}
\hline \multicolumn{3}{|l|}{ Table I. The laboratory values of the patient at the diagnosis } \\
\hline Parameters & $\begin{array}{l}\text { Patient } \\
\text { value }\end{array}$ & $\begin{array}{l}\text { Normal } \\
\text { range }\end{array}$ \\
\hline Glucose (mg/dL) & 137 & $60-100$ \\
\hline Total cholesterol (mg/dL) & 137 & $<170$ \\
\hline Triglyceride (mg/dL) & 38 & $<150$ \\
\hline LDL-cholesterol (mg/dL) & 87 & $<130$ \\
\hline HDL-cholesterol(mg/dL) & 42 & $>45$ \\
\hline C-peptide (ng/mL) & 1.66 & $0.9-7.1$ \\
\hline Hemoglobin (gr/dL) & 12.8 & $12-15.6$ \\
\hline Glycated hemoglobin (HbA1c) (\%) & 8.8 & $4-6.0$ \\
\hline Anti-thyroid peroxidase (IU/mL) & 0.9 & $0-9$ \\
\hline Anti-thyroglobulin (IU/mL) & 1.1 & $0-4$ \\
\hline Anti-tissue transglutaminase (U/mL) & 1.8 & $0-20$ \\
\hline Anti-insulin antibody (\%) & 6.2 & $<8.2$ \\
\hline Glutamic acid decarboxylase (IU/mL) & 0.26 & $0-10$ \\
\hline Islet cell antibody & Negative & Negative \\
\hline
\end{tabular}

HDL: High-density lipoprotein, LDL: Low-density lipoprotein and HbA1c levels. These findings indicated a most probable diagnosis of MODY. We initiated an insulin glargine only treatment ( 0.2 unit $/ \mathrm{kg} /$ day). Postprandial hyperglycemia was rarely observed and no significant hypoglycemia was seen with this treatment. HbA1c decreased to $6.3 \%$.

\section{Molecular Analysis}

Genomic DNA was extracted from peripheral blood leukocytes of the patient and her parents by using MagNA Pure LC DNA Isolation Kit I (Roche Diagnostics, Mannheim, Germany) according to the manufacturer's protocol. All coding exons and exon-intron boundaries of the HNF4A gene were amplified by polymerase chain reaction (PCR). After purification of PCR products, mutational analysis was performed by direct sequencing of the coding exons and flanking introns of the HNF4A gene in an ABI PRISM 3500 genetic analyzer (Applied Biosystems, Foster City, California, USA). As a reference sequence, NM_175914 (obtained from GenBank accession number) for HNF4A was used. While the father and mother had no mutation, analysis of the patient revealed a p.C93Y (c.278G >A) heterozygous novel change in the third exon of HNF4A (Figure 1). This missense mutation was not found in the Ensembl and Human Gene Mutation Database (HGMD). It was interpreted to be "disease causing" by the Mutation Taster Software (test score: 0.999999999999997) (http://www.mutationtaster.org). The cysteine residue in position 93 is highly conserved across different species (Figure 2). Insulin treatment was stopped and low-dose sulfonylurea ( $5.0 \mathrm{mg} /$ day in two doses) initiated as soon as the diagnosis of MODY 1 was made. After five months of the administering, glucose monitoring was within

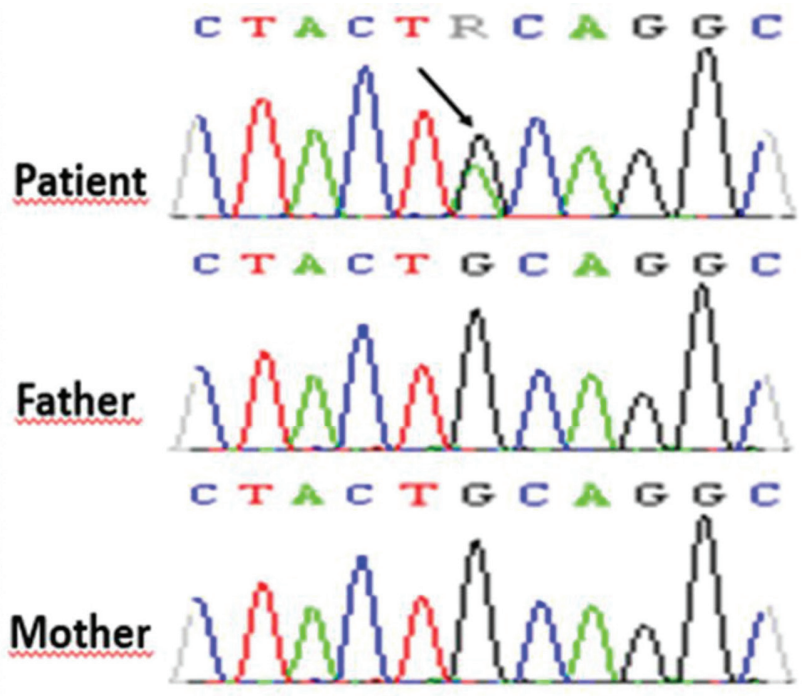

Figure 1. Partial sequence traces for the HNF4A gene of the father, mother and patient. Analysis of the patient revealed heterozygous and de novo a G-to-A ( $R=A$ ) substitution (c.278G $>A$ ) that changes cysteine to tyrosine amino acid (p.C93Y) in exon 3 


\begin{tabular}{|c|c|c|}
\hline Iranscript IDs & Species & Amino acid alignments around position 93 \\
\hline ENSTO0000316673 & Human (Homo sapiens) & _-_VDKDKRNQCRY C RLKKCFRAGMK... \\
\hline ENSPTRG00000013519 & Chimpanzee (Pan troglodytes) & _-VDKDKRNQCRY C RLKKCFRAGMK... \\
\hline ENSMMUG00000006464 & Monkey (Macaca mulatta) & .-.VDKDKRNQCRY C RLKKCFRAGMK... \\
\hline ENSFCAG00000008178 & Cat (felis catus) & _-VDKDKRNQCRY C RLKKCFRAGMK... \\
\hline ENSMUSG00000017950 & House Mouse (Mus musculus) & _-VDKDKRNQCRY C RLKKCFRAGMK... \\
\hline ENSGALG00000004285 & Chicken (Gallus gallus) & .-.VDKDKRNQCRY C RLKKCFRAGMK... \\
\hline ENSTRUG00000009982 & Pufferfish (Takifugu rubripes) & _.VDKOKRNQCRY C RLKKCFRAGMK... \\
\hline ENSDARG00000021494 & Zebrafish (Danio rerio) & _-VDKDKRNQCRY C RLKKCFRAGMK... \\
\hline $\mathrm{T} 23 \mathrm{H} 4.2$ & $\begin{array}{l}\text { Nematode (Caenorhabditis } \\
\text { elegans) }\end{array}$ & ..VTKNKKRNACRA C RLQKCVKAGMK... \\
\hline ENSXETGO00000001775 & Frog (Xenopous tropicalis) & _-VDOKDKRNQCRY C RLKKCFRAGMK... \\
\hline
\end{tabular}

Figure 2. Partial protein alignment of HNF4A gene from different species around position 93 . The cysteine residue in position 93 is highly conserved. Cystein is a polar neutral amino acid whereas tyrosine is a polar hydrophilic amino acid. C93Y mutation may change the secondary or tertiary structure of HNF4A protein and impair its function

the normal range during sulfonylurea treatment and no hypoglycemia was observed. Laboratory evaluation revealed fasting glucose at $111 \mathrm{mg} / \mathrm{dL}$, insulin at $11 \mathrm{IU} / \mathrm{mL}$, C-peptide at $2.2 \mathrm{ng} / \mathrm{mL}$ and HbA1c at $5.8 \%$.

\section{Discussion}

CCK and HNF1A mutations are responsible for the majority of MODY cases. Of all MODY cases, $20-50 \%$ are caused by GCK and HNF1A, approximately $10 \%$ are from a mutation of HNF4A or HNF1B (3). Studies from our country reported that GCK is the most common subtype (9-11). In the present study, we have identified a novel heterozygous G-to-A substitution at 278 position (c.278G $>A$ ) that changes cysteine to tyrosine amino acid (p.C93Y) in exon 3 in HNF4A, which leads to MODY Type I. Flanagan et al. (8) have reported a different de novo HNF4A mutation at the same position (p.C93S, c.278 $\mathrm{G}>\mathrm{C}$ ) leading to a diazoxide responsive hyperinsulinemic hypoglycemia that was diagnosed within the first week of life in a patient born with macrosomia (4.100 gr). Our group (9) did not detect HNF4A mutations in 42 children diagnosed with MODY, but Ağladıoğlu et al. (10) analyzed 43 patients with MODY and identified two cases with the same heterozygous HNF4A mutations. One of the cases had a missense mutation (c.416C > T), which is associated with Type Il diabetes mellitus in the HGMD. The other patient was carrying both heterozygous HNF4A (c.416C>T) and HNF1A mutations. As distinct from those two cases, our case had a de novo mutation. Findings of the multicenter study by Stanik et al. (4) underlined that de novo mutations of cases with MODY are more frequent than previously assumed. As a result, the authors emphasize the importance of genetic testing for MODY in patients without a family history (4). Thanabalasingham et al. (12) reported that measurable serum C-peptide is valuable in the diagnosis of MODY in individuals diagnosed with diabetes before 30 years of age. Moreover, they speculated that a family history of diabetes, presence or absence of autoantibodies regarding diabetes and metabolic disturbances (e.g. insulin resistance) were less important than previously thought (13). In the present case, the age at onset of diabetes, the negative family history, normal BMI, an absence of autoantibodies to pancreatic cell fragments and a measurable C-peptide level indicated MODY and a novel mutation in HNF4A was detected subsequently. Consistent with the literature, this patient was highly responsive to even low doses of sulfonylurea with no hypoglycemia. The majority of MODY Type I individuals are born with macrosomia (>4.000 gr) similar to the offspring of women with diabetes (13). Macrosomia is related to considerable fetal and maternal morbidity (13). In case of maternal diabetes, incremental glucose exposure to the fetus via the placenta results in incremental fetal insulin secretion and macrosomia develops subsequently due to insulinmediated growth. However, the mother is normoglycemic in cases of de novo heterozygous HNF4A mutations and 
associated fetal macrosomia is thought to be related to a different, yet unknown mechanism. HNF4A mutations are thought to have dual opposite roles in insulin secretion from beta cells (13). While these mutations usually lead to increased insulin secretion and subsequent hypoglycemia in newborns (not seen in our case), this effect is switched to impaired insulin secretion in adulthood resulting in glucose intolerance. Pearson et al. (5) asserted the underlying etiology of macrosomia in cases with a HNF4A mutation is associated with incremental endogenous insulin production. Since they found that $56 \%$ of the newborns with a heterozygous HNF4A mutation were macrosomic, birth weight was considered to be related with individual genetic characteristics as well as the maternal intra-uterine environment (6). All in all, HNF4A gene mutation should be considered in differential diagnosis of macrosomic newborns in spite of a negative family history for diabetes or hypoglycemia. Unlike MODY 2 cases, progressive hyperglycemia becomes evident in individuals of MODY 1 and MODY 3. Therefore, besides dietary treatment, they frequently require pharmacotherapy such as sulfonylureas, which usually allows for better glycemic control especially in children and young adults $(4,7,14)$. It is well known that MODY 1 and MODY 3 cases are likely to develop microvascular complications at a similar rate compared with those of Type I or II diabetes (14). We switched the treatment of our case from insulin to sulfonylurea (glibenclamide $5 \mathrm{mg} /$ day, b.i.d) when the diagnosis of MODY 1 was genetically proven. In followup, glycemic control was better and no hypoglycemia was observed. The transcription factor of HNF4A is an activator of genes involved in the control of lipid homeostasis as well as glucose metabolism (15). It has been demonstrated that HNF4A mutation carriers have low circulating triglycerides and apolipoprotein concentrations (16). In line with this, in another study, it was shown that a HNF4A knockout mouse had reduced fasting serum levels of total cholesterol, high-density lipoprotein, triglycerides and apolipoprotein (16). Our patient, similarly, had low serum level of triglycerides, which suggests an essential role of HNF4A in the complex transcription factor network that controls lipid regulation. In conclusion, herein, we described a rare cause of diabetes resulting from a novel and de novo heterozygous mutation in the HNF4A gene. We emphasize that genetic testing is crucial for both establishing an accurate diagnosis and providing an option to determine whether patients are sensitive to sulfonylurea or not. In addition, we underline that genetic testing of HNF4A might be considered for carefully selected patients born with macrosomia without hypoglycemia or a family history of diabetes.

\section{Acknowledgements}

This research did not receive any specific grant from any funding agency in the public, commercial or not-for-profit sector.

\section{Ethics}

Informed Consent: Consent form was obtained from the patient and her parents.

Peer-review: External and internal peer-reviewed.

\section{Authorship Contributions}

Surgical and Medical Practices: S.A., A.A., E.B., Concept: S.A., K.D., T.R.Ö., Design: S.A., B.Ö., A.A., Data Collection or Processing: S.A., K.D., T.R.Ö., Analysis or Interpretation: S.A., A.A., E.B., Literature Search: K.D., B.Ö., Writing: S.A., A.A., K.D.

Conflict of Interest: No conflict of interest was declared by the authors.

Financial Disclosure: The authors declared that this study has received no financial support.

\section{References}

1. Chambers C, Fouts A, Dong F, et al. Characteristics of maturity onset diabetes of the young in a large diabetes center. Pediatr Diabetes 2016;17:360-7.

2. Shepherd M, Ellis I, Ahmad AM, et al. Predictive genetic testing in maturity-onset diabetes of the young (MODY). Diabet Med 2001;18:417-21.

3. Ellard S, Bellanné-Chantelot C, Hattersley AT; European Molecular Genetics Quality Network (EMQN) MODY group. Best practice guidelines for the molecular genetic diagnosis of maturity-onset diabetes of the young. Diabetologia 2008;51:546-53.

4. Stanik J, Dusatkova $P$, Cinek $O$, et al. De novo mutations of GCK, HNF1A and HNF4A may be more frequent in MODY than previously assumed. Diabetologia 2014;57:480-4.

5. Pearson ER, Boj SF, Steele AM, et al. Macrosomia and hyperinsulinaemic hypoglycaemia in patients with heterozygous mutations in the HNF4A gene. PLoS Med 2007;4:e118.

6. Roženková K, Güemes M, Shah P, Hussain K. The Diagnosis and Management of Hyperinsulinaemic Hypoglycaemia. I Clin Res Pediatr Endocrinol 2015;7:86-97.

7. Pearson ER, Pruhova S, Tack C), et al. Molecular genetics and phenotypic characteristics of MODY caused by hepatocyte nuclear factor 4alpha mutations in a large European collection. Diabetologia 2005;48:878-85.

8. Flanagan SE, Kapoor RR, Mali G, et al. Diazoxide-responsive hyperinsulinemic hypoglycemia caused by HNF4A gene mutations. Eur J Endocrinol 2010;162:987-92.

9. Anık A, Çatlı G, Abacı A, et al. Molecular diagnosis of maturityonset diabetes of the young (MODY) in Turkish children by using targeted next-generation sequencing. I Pediatr Endocrinol Metab 2015;28:1265-71.

10. Ağladıoğlu SY, Aycan Z, Çetinkaya S, et al. Maturity onset diabetes of youth (MODY) in Turkish children: sequence analysis of 11 causative genes by next generation sequencing. I Pediatr Endocrinol Metab 2016;29:487-96.

11. Haliloglu B, Hysenaj G, Atay Z, et al. GCK gene mutations are a common cause of childhood-onset MODY (maturityonset diabetes of the young) in Turkey. Clin Endocrinol (Oxf) 2016;85:393-9. 
12. Thanabalasingham G, Pal A, Selwood MP, et al. Systematic assessment of etiology in adults with a clinical diagnosis of young-onset type 2 diabetes is a successful strategy for identifying maturity-onset diabetes of the young. Diabetes Care 2012;35:1206-12.

13. Chauhan SP, Grobman WA, Gherman RA, et al. Suspicion and treatment of the macrosomic fetus: a review. Am J Obs Gynecol 2005;193:332-46.

14. Rubio-Cabezas O, Hattersley AT, Niølstad PR, et al. ISPAD Clinical Practice Consensus Guidelines 2014. The diagnosis and management of monogenic diabetes in children and adolescents. Pediatr Diabetes 2014;15(Suppl 20):47-64.

15. Pearson ER, Pruhova S, Tack C), et al. Molecular genetics and phenotypic characteristics of MODY caused by hepatocyte nuclear factor 4alpha mutations in a large European collection. Diabetologia 2005;48:878-85.

16. Hayhurst GP, Lee $\mathrm{YH}$, Lambert G, Ward IM, Gonzalez Fl. Hepatocyte nuclear factor 4alpha (nuclear receptor 2A1) is essential for maintenance of hepatic gene expression and lipid homeostasis. Mol Cell Biol 2001;21:1393-403. 